\title{
1 Temporal cascade of frontal, motor and muscle processes underlying human
}

\section{2 action-stopping}

3

4 Sumitash Jana * 1, Ricci Hannah * 1, Vignesh Muralidharan ${ }^{1}$, and Adam R. Aron ${ }^{1}$ 5

$6 *$ These authors contributed equally to the study

7

$8 \quad{ }^{1}$ Department of Psychology, University of California, San Diego

9

10 Materials \& Correspondence

11 Correspondence and requests should be addressed to S.J. (s2jana@ucsd.edu) or to R.H.

12 (rhannah@ucsd.edu)

13

14 


\section{Abstract}

Action-stopping is a canonical executive function thought to involve top-down control over the motor system. Here we aimed to validate this stopping system using high temporal resolution methods in humans. We show that, following the requirement to stop, there was an

19 increase of right frontal beta $(\sim 13$ to $30 \mathrm{~Hz})$ at $\sim 120 \mathrm{~ms}$, likely a proxy of right inferior frontal

20 gyrus; then, at $140 \mathrm{~ms}$, there was a broad skeletomotor suppression, likely reflecting the impact

21 of the subthalamic nucleus on basal ganglia output; then, at $\sim 160 \mathrm{~ms}$, suppression was detected

22 in the muscle, and, finally, the behavioral time of stopping was $\sim 220 \mathrm{~ms}$. This temporal cascade

23 confirms a detailed model of action-stopping, and partitions it into subprocesses that are isolable

24 to different nodes and are more precise than the behavioral speed of stopping. Variation in these

25 subprocesses, including at the single-trial level, could better explain individual differences in

26 impulse control.

The ability to control one's actions and thoughts is important for our daily lives; for

30 example: changing gait when there is an obstacle in the path ${ }^{1}$, resisting the temptation to eat

31 when on a diet ${ }^{2}$, overcoming the tendency to say something hurtful ${ }^{3}$. While many processes

32 contribute to such forms of control, one important process is response inhibition - the prefrontal

33 (top-down) stopping of initiated response tendencies ${ }^{4}$. In the laboratory, response inhibition is

34 often studied with the stop-signal task ${ }^{5}$. On each trial, the participant initiates a motor response,

35 and then, when a subsequent Stop signal occurs, tries to stop. From the behavioral data one can

36 estimate a latent variable; the speed of stopping known as Stop Signal Reaction Time (SSRT),

37 which is typically $200-250 \mathrm{~ms}$ in healthy adults ${ }^{5}$. SSRT has been useful in neuropsychiatry

38 where it is often longer for patients vs. controls ${ }^{6-11}$. The task has also provided a rich test-bed, 
across species, for mapping out a putative neural architecture of prefrontal-basal-ganglia-regions

40 for rapidly suppressing motor output areas ${ }^{6,12,13}$. Given this rich literature, this task is one of the

41 few paradigms included in the longitudinal Adolescent Brain Cognitive Development study ${ }^{14}$ of

4210,000 adolescents over 10 years.

Against this background, a puzzle is that the relation between SSRT and 'real-world'

44 self-reported impulsivity is often weak ${ }^{15-20}$. One explanation is that SSRT may not accurately

45 index the brain's true stopping speed. Indeed, recent mathematical modelling of behavior during

46 the stop-signal task suggests that standard calculations of SSRT may overestimate the brain's

47 stopping speed by $\sim 100 \mathrm{~ms}^{15}$ [also see ${ }^{21}$. Further, in a recent study ${ }^{22}$, electromyographic (EMG)

48 recordings revealed an initial increase in EMG activity in response to the Go cue, followed by a

49 sudden decline at $\sim 150 \mathrm{~ms}$ after the Stop signal. This decline in EMG could be because of the

50 Stop process 'kicking in' to cancel motor output - but the striking thing is that this was $50 \mathrm{~ms}$

51 before the SSRT of $200 \mathrm{~ms}$. This timing is also consistent with experiments using transcranial

52 magnetic stimulation (TMS) to measure the motor evoked potential (MEP) during the stop-signal

53 task (the MEP indexes the excitability of the pathways from motor cortex to muscle). The MEP

54 in the muscle that was-to-be-stopped reduced at $\sim 150 \mathrm{~ms}^{23,24}$. Further, other studies that

55 measured the MEP from muscles that were not needed for the task, show there is 'global

56 suppression' also at $\sim 150 \mathrm{~ms}^{25-28}$ (i.e. corticospinal activity was suppressed for the broader

57 skeletomotor system). This 'global MEP suppression' has been linked to activation of the

58 subthalamic nucleus of the basal-ganglia ${ }^{29}$, which is thought to be critical for stopping, and might

59 broadly inhibit thalamocortical drive ${ }^{30}$.

The potential overestimation of the brain's true stopping speed by SSRT could arise for

61 several reasons. First, the race model assumes that the Stop process is "triggered" on every trial. 
62 But recent research shows that this is not the case ${ }^{15}$, and that failing to account for "trigger

63 failures" inflates SSRT. Second, while the standard "race model" assumes that the Go and Stop

64 processes are independent ${ }^{5}$, recent research show that violations of this independence can also

65 inflate SSRT ${ }^{21}$. Finally, the standard ways of computing SSRT likely do not account for

66 electromechanical delays between muscle activity and the response. In any event, overestimating

67 the brain's stopping speed would add variance to SSRT which could potentially weaken the

68 above-mentioned across-participant associations between stopping speed and self-report

69 scores ${ }^{15-17}$. Furthermore, if the true stopping speed is $\sim 150 \mathrm{~ms}$, the timing of activation of nodes

70 in the putative response inhibition network should precede this time-point for those nodes to play

71 a causal role in action stopping - and this is important for the interpretation of neuroscience

72 studies. For instance, in electrocorticography and electroencephalography (EEG) studies,

73 successful stopping elicits increased beta band power over right frontal cortex in the time period

74 between the Stop signal and SSRT ${ }^{31-33}$. Whether this, and other, neurophysiological markers of

75 the Stop process occur sufficiently early to directly contribute to action-stopping (if SSRT is

76 overestimated) is unknown; yet this is fundamental to our understanding of brain networks

77 underlying response inhibition.

Here we leveraged the insight from the above-mentioned study ${ }^{22}$ which used EMG of the

79 task relevant muscles. We now tested whether we could derive a single trial estimate of stopping

80 speed from EMG (referred to as CancelTime). More specifically, we hypothesized that 'partial'

81 EMG bursts on the Successful Stop trials (i.e. small EMG responses that begin but do not reach a

82 sufficient amplitude to lead to an overt response) $)^{34}$ would carry information about the latency of

83 stopping and tested this in two studies. In a third study we tested if CancelTime would

84 correspond with the measure of putative basal ganglia-mediated global motor suppression, 
85 measured with single-pulse TMS. In studies four and five we turned to the cortical process

86 thought to initiate action-stopping, using the above-mentioned proxy of right frontal beta ${ }^{31,32}$.

87 We measured scalp EEG, derived a right frontal spatial filter in each participant, and then

88 extracted beta bursts ${ }^{35}$ in the time period between the Stop signal and SSRT. We tested how the

89 timing of these beta bursts related to CancelTime.

90

91 Results

92 Study 1 (EMG). 10 participants performed the stop-signal task (Fig. 1a). On each trial they

93 initiated a manual response when a Go cue occurred, and then had to try to stop when a Stop

94 signal suddenly appeared on a minority of trials. Depending on the stop signal delay, SSD,

95 participants succeeded or failed to stop, each $\sim 50 \%$ of the time). We measured EMG from the

96 responding right index and little fingers (Fig. 1b inset). Behavioral performance was typical, with

97 SSRT (referred to as $\mathrm{SSRT}_{\mathrm{Beh}}$ ) of $216 \pm 8 \mathrm{~ms}$, and action-stopping on $51 \pm 1 \%$ of Stop trials (Table

98 1). EMG analysis was performed on the trial-by-trial root-mean-squared EMG (EMGRMs; Fig.

99 1b). On $53 \pm 6 \%$ of Successful Stop trials (i.e. where no keypress was made) there was a small

100 but detectible EMG response (Partial EMG trials; see Supplementary Fig. 1a, b for EMG-RT

101 correlation), while on the remainder of Successful Stop trials there was no detectible EMG

102 response (No EMG trials). The amplitude of EMG responses (mean peak EMG voltage) in the

103 Partial EMG trials was $48 \pm 3 \%$ smaller than in trials with a keypress (Fig. 2a). 

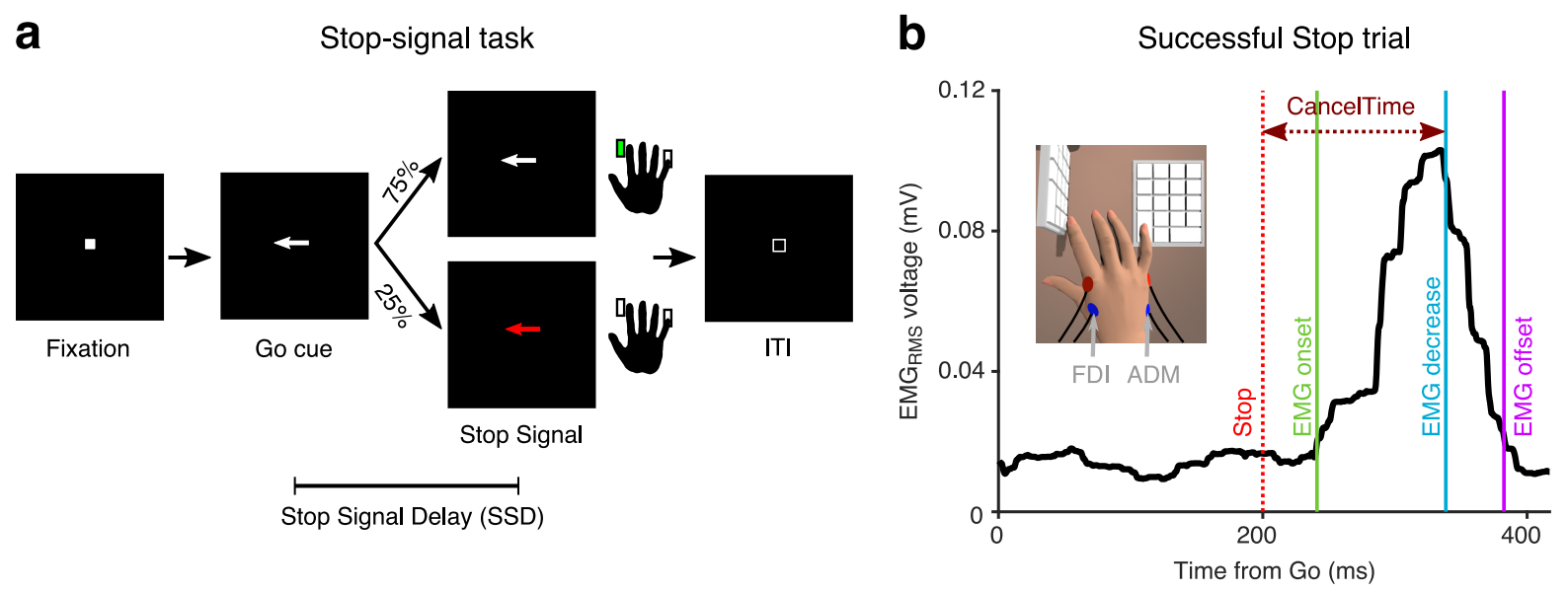

Figure 1 | Behavioral task and EMG recording. (a) Stop-signal task. (b) EMG $\mathrm{RMS}_{\mathrm{RMS}}$ on a Successful

Stop trial (Partial EMG) in an exemplar participant, aligned to the Go cue. CancelTime refers to the time from the Stop signal (dotted red line) to when the $\mathrm{EMG}_{\mathrm{RMS}}$ starts decreasing (blue line). The green and purple line represent the detected onset and offset of the EMG response. (Inset) Recording set-up 109

110 Table 1: Behavior (mean \pm s.e.m.; All values in ms)

\begin{tabular}{llllll} 
& $\begin{array}{l}\text { Study 1 } \\
\text { (EMG) }\end{array}$ & $\begin{array}{l}\text { Study 2 } \\
\text { (EMG) }\end{array}$ & $\begin{array}{l}\text { Study 3 } \\
\text { (TMS) }\end{array}$ & $\begin{array}{l}\text { Study 4 } \\
\text { (EEG) }\end{array}$ & $\begin{array}{l}\text { Study 5 } \\
\text { (EEG) }\end{array}$ \\
\hline Go RT & $470(15)$ & $493(15)$ & $430(17)$ & $427(15)$ & $405(6)$ \\
Failed Stop RT & $416(11)$ & $447(14)$ & $391(12)$ & $384(12)$ & $370(5)$ \\
Correct Go \% & $97(1)$ & $98(0)$ & $99(0)$ & $99(0)$ & $99(0)$ \\
Correct Stop \% & $51(1)$ & $52(1)$ & $49(1)$ & $48(1)$ & $50(0)$ \\
Mean SSD $_{\text {SSRT }}$ Beh & $237(20)$ & $280(17)$ & $194(18)$ & $191(21)$ & $170(7)$ \\
S & $216(8)$ & $204(4)$ & $219(6)$ & $214(9)$ & $219(6)$
\end{tabular}

113 Stop signal is a readout of the time when the Stop process is implemented in the muscle

114 (hereafter 'CancelTime'). We observed that, first, CancelTime is much earlier than SSRT Beh $_{\text {(see }}$

115 Fig. 2c (left) for all CancelTimes in an exemplar participant; mean CancelTime $=146 \pm 3 \mathrm{~ms}$, 
$116 \mathrm{SSRT}_{\mathrm{Beh}}=203 \mathrm{~ms}$ ); and second, across participants, CancelTime was positively correlated with

117 SSRT $_{\text {Beh }}$ (Fig. 2d; study 1: mean CancelTime $=152 \pm 11 \mathrm{~ms}$, mean SSRT $\mathrm{Beh}=216 \pm 8 \mathrm{~ms} ; r=$

$\left.1180.71, p=0.020, B F_{10}=3.6\right)$. This suggests that CancelTime might index the time when Stopping

119 is implemented at the muscle.

120

a

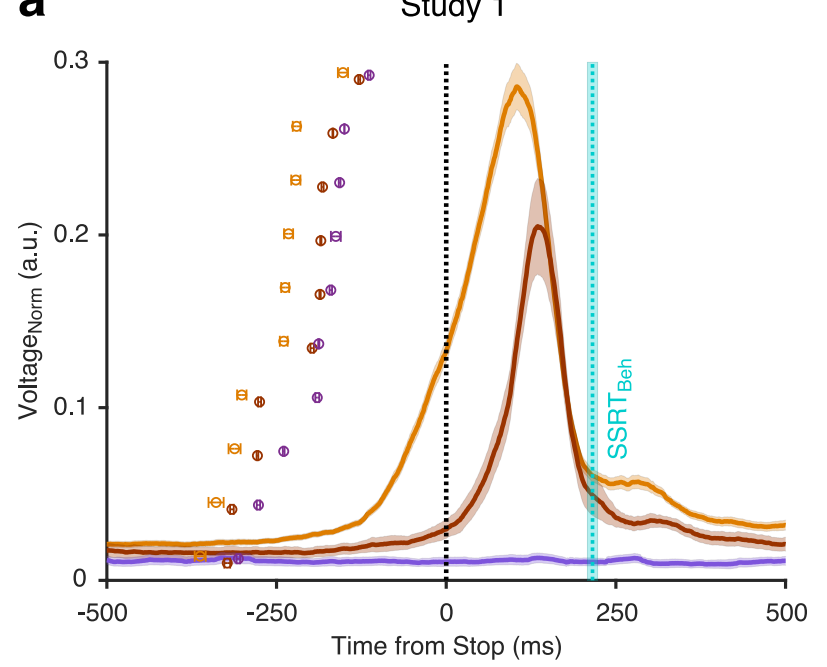

b Study 2

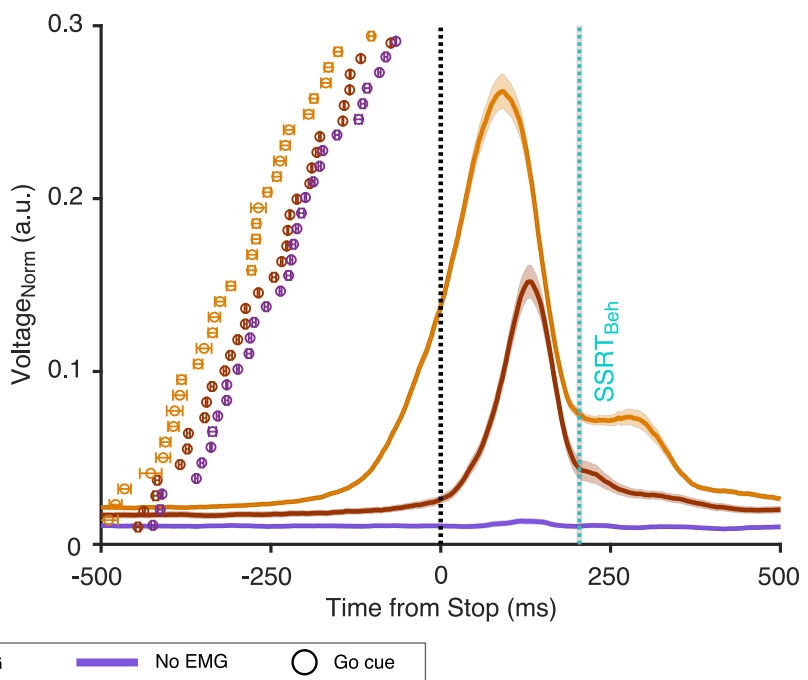

d

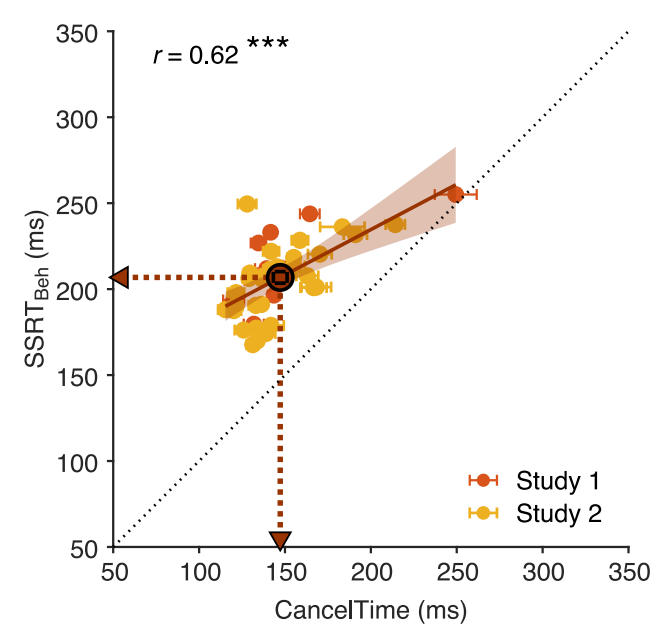

C

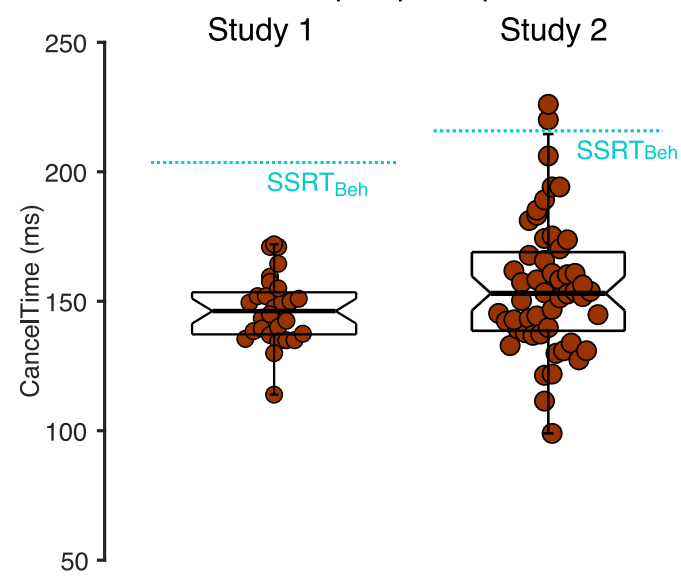

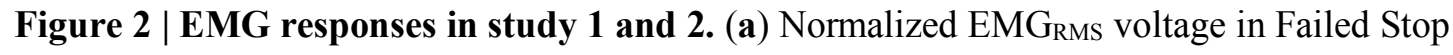


represent the mean \pm s.e.m of $\mathrm{SSRT}_{\mathrm{Beh}}$ across participants. The dots and cross-hairs represent the

mean \pm s.e.m. of the Go cue in a participant. Note that the time between the Go cue and the Stop signal

(i.e. the SSD) is shortest for the No EMG (purple), then the Partial EMG (brown), and then the Failed

Stop trials (orange). (b) Same as (a) but for Study 2. (c) (Right) Beeswarm plot of the CancelTime in an exemplar participant from Study 1. Each dot represents a trial. The dotted cyan line represents the SSRT $_{\text {Beh. }}$ (Left) Same as right but for Study 2. (d) Correlation between CancelTime and SSRT Beh in $_{\text {in }}$

134 Study 2 (EMG). We then ran a new sample ( $n=32$; see Table 1 for behavioral results). Again, 135 we observed partial EMG responses on $49 \pm 2 \%$ of Successful Stop trials; where the EMG 136 amplitude was $54 \pm 1 \%$ smaller than the amplitude in trials with a keypress (Fig. 2b). Fig. 2c

137 (right) shows the distribution of CancelTimes in an exemplar participant (mean CancelTime $=$

$\left.138156 \pm 4 \mathrm{~ms}, \mathrm{SSRT}_{\mathrm{Beh}}=218 \mathrm{~ms}\right)$. Again, across participants, mean CancelTime was positively

139 correlated with SSRT ${ }_{\text {Beh }}$ (Fig. 2d; mean CancelTime $=146 \pm 4$ ms, mean SSRT Beh $=204 \pm 4 \mathrm{~ms} ; r$

$\left.140=0.59, p<0.001, B F_{10}=71.7\right)$. Intriguingly, in each study, CancelTime was $\sim 60 \mathrm{~ms}$ less than

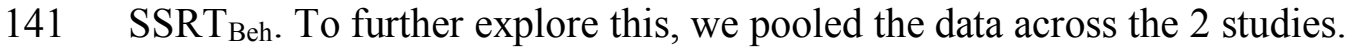

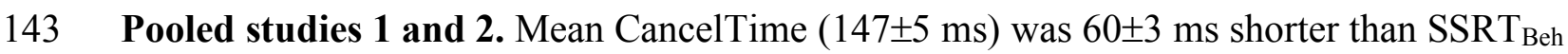

$144\left(t(41)=18.4, p<0.001, d=2.5, B F_{10}>100\right)$. We then tested whether we could calculate SSRT

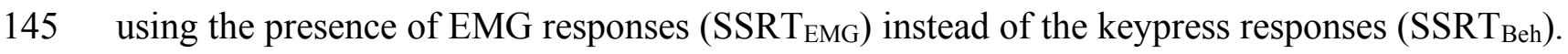

146 We considered Partial EMG trials as Failed Stop trials and used EMG onset time on Correct Go 147 trials to recalculate SSRT (i.e. instead of using P(Respond|Stop) from behavior and Go RT as is 
148 typical for SSRT $_{\text {Beh }}$ calculations; see Methods; see Fig. 3a for an exemplar participant). We then

149 performed 1-way repeated measures ANOVA with "Stop Time" as the dependent measure and

150 the method of estimation as a factor $\left(\mathrm{SSRT}_{\mathrm{EMG}}, \mathrm{SSRT}_{\mathrm{Beh}}\right.$, and CancelTime). There was a

151 significant main effect of the estimation method on "Stop Time" $\left(F_{G G}(1.4,56.1)=66.3, p<\right.$

$\left.1520.001, \eta_{p}^{2}=0.6\right)$. Pairwise comparisons showed that $\operatorname{SSRT}_{\mathrm{EMG}}(157 \pm 7 \mathrm{~ms})$ was significantly

153 faster than $\operatorname{SSRT}_{\text {Beh }}(209 \pm 3 \mathrm{~ms})\left(\right.$ Fig. $\left.3 \mathrm{~b} ; t(41)=8.2, p_{\text {Bon }}<0.001, d=1.3, B F_{10}>100\right)$, but

154 importantly, not significantly different from mean CancelTime $\left(t(41)=1.5, p_{B o n}=0.270, d=0.2\right.$,

$\left.155 B F_{10}=0.5\right)$. This suggests that $\mathrm{SSRT}_{\mathrm{Beh}}$ might be protracted by a peripheral delay and that

156 CancelTime might be a better metric of the time of implementation of the Stop process.

Next, we examined in more detail the EMG profile on Partial EMG trials. Across all

158 participants, the EMG response in the Partial EMG trials (when aligned to the EMG onset) had a

159 profile similar to the EMG response in the Correct Go and Failed Stop trials, but diverged $\sim 55$

$160 \mathrm{~ms}$ after EMG onset (55 ms compared to Correct Go, and $56 \mathrm{~ms}$ compared to Failed Stop trials,

161 Fig. 3c). We surmised that if the Partial EMG trials reflect responses that have been actively

162 cancelled at the muscle-level, then the amplitude of these responses should increase with SSD.

163 The rationale was that, at shorter SSDs, the Go process will have been active for a shorter

164 duration, meaning EMG activity will not have increased much before being inhibited, while at

165 longer SSD, the Go process will have been active for a longer duration, meaning EMG activity

166 will have increased much more before being inhibited. Indeed, the amplitude of the Partial EMG

167 responses increased with SSD (Supplementary Fig. 1c). A 1-way repeated measures ANOVA

168 with amplitude as the dependent variable and the SSD as the independent variable showed

169 significant effect of SSD on amplitude $\left(F(4,24)=3.7, p=0.018, \eta_{p}^{2}=0.4\right)$ [also see ${ }^{23}$. This 
170 suggests that the Partial EMG trials represent inhibited Go responses and not merely a weak Go

171 process (which would presumably not increase across SSDs).

172 To further validate CancelTime, we modelled the behavior using BEESTS (Bayesian

173 Estimation of Ex-gaussian STop-Signal reaction time distributions; see Table 2 for model

174 estimates). While $\mathrm{SSRT}_{\text {Beh }}$ produces a single estimate per person, BEESTS uses a Bayesian

175 parametric approach to estimate the distribution of SSRTs ${ }^{36}$. Also, for each participant, it

176 provides an estimate of the probability of trigger failures (i.e. stop trials where the stopping

177 process was not initiated $\left.{ }^{36}\right)$. Across participants, mean CancelTime was positively correlated

178 with the mean $\operatorname{SSRT}_{\text {BEESTS }}\left(205 \pm 3 \mathrm{~ms} ; r=0.54, p<0.001, B F_{10}>100\right.$; Fig. 3d). More

179 interestingly, the SD of CancelTime $(33 \pm 2 \mathrm{~ms})$ was positively correlated with the SD of

$180 \operatorname{SSRT}_{\text {BEESTS }}\left(48 \pm 5 \mathrm{~ms} ; r=0.42, p=0.005, B F_{10}=6.9\right.$; Fig. 3e $)$. Further, the percentage of trigger

181 failures $(4 \pm 1 \%)$ was positive correlated with mean CancelTime $\left(\rho=0.57, p<0.001, B F_{10}>100\right)$

182 suggesting that participants who fail to "trigger" the Stop process more often, have longer

183 CancelTimes (Fig. 3f). These relationships between CancelTime and model estimates give

184 further credence to our interpretation that CancelTime on Partial EMG trials reflects a single-trial 185 measure of the time of implementation of the Stop process. 

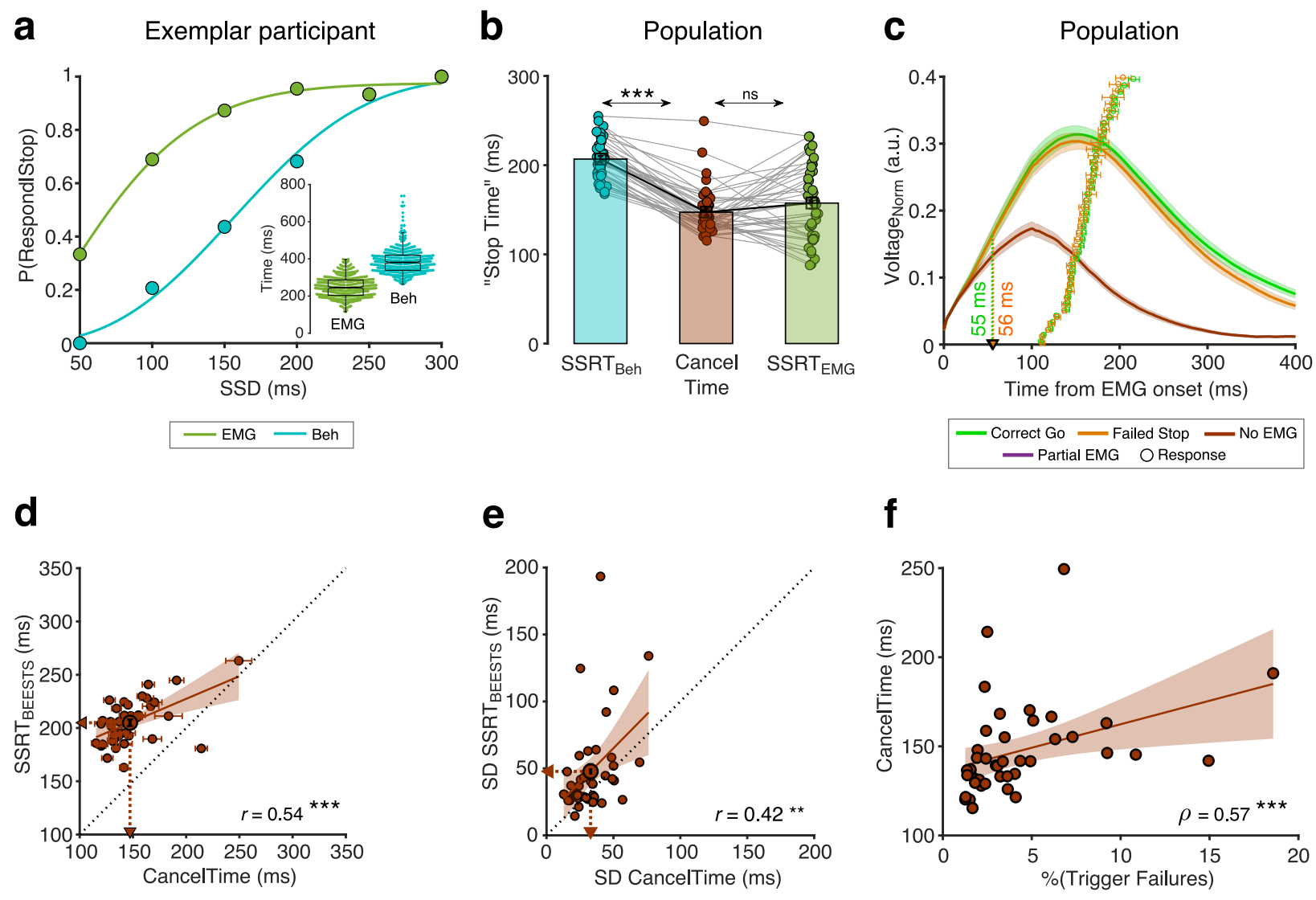

Figure 3 | Peripheral delay associated with SSRT $_{\text {Beh }}$ and relationship between CancelTime and

BEESTS parameters. (a) $\mathrm{P}($ Respond|Stop) in an exemplar participant calculated using the behavioral response (dark green dots) and the EMG response (cyan dots). The lines represent the cumulative Weibull fit as $W(t)=\gamma-(\gamma-\delta) e^{\left[-(t / \alpha)^{\beta}\right]}$ where where $t$ is the $\mathrm{SSD}, \alpha$ is the time at which the function reaches $64 \%$ of its full growth, $\beta$ is the slope, $\delta$ is the minimum value of the function, and $\gamma$ is maximum value of the function. The difference between $\delta$ and $\gamma$ marks range of the function.

(Inset) Beeswarm plot of the EMG onset (dark green) and the behavioral responses (cyan) used to calculate $\mathrm{SSRT}_{\mathrm{EMG}}$ and $\mathrm{SSRT}_{\mathrm{Beh}}$ respectively. (b) Comparison of the $\mathrm{SSRT}_{\mathrm{Beh}}$ (cyan), CancelTime (brown), and $\operatorname{SSRT}_{\mathrm{EMG}}($ dark green) across all participants. Each dot represents a participant, while the bar and cross-hair represents the mean \pm s.e.m. in a group. (c) The normalized EMG responses aligned to the detected EMG onsets in the Correct Go (dark green), Failed Stop (orange), and Partial EMG (brown) trials. The line and shaded region represent the mean \pm s.e.m. in a group. The dots and cross 
hairs represent the mean \pm s.e.m. of the keypress in a participant. (d) Correlation between CancelTime

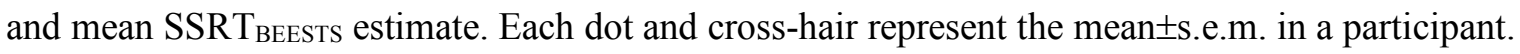
$\mathrm{SD}$ of the $\mathrm{SSRT}_{\mathrm{BEESTS}}$ estimate. Other details same as (d). (f) Correlation between percentage Trigger Failures estimated from BEESTS and CancelTime. Other details same as (d).

Table 2: BEESTS estimates (mean \pm s.e.m.; All values in $\mathrm{ms}$ )

\begin{tabular}{|ll|}
\hline Estimated parameters & Pooled study 1 \& 2 \\
\hline Mean Go RT & $483(13)$ \\
\hline SD Go RT & $94(5)$ \\
Mean SSRT & $205(3)$ \\
SD SSRT & $48(5)$ \\
\%Trigger Failures & $4(1)$
\end{tabular}

221 Study 3 (TMS). To further validate CancelTime and relate it to brain processes we turned to a

222 different method - single-pulse TMS over a task-irrelevant muscle representation in the brain. As

223 mentioned above, the reduction of MEPs from task-irrelevant muscles on Successful Stop

224 trials $^{25-27}$, is thought to reflect a basal ganglia-mediated global suppression ${ }^{29}$. Eighteen new

225 participants (see Table 1 for behavioral results) now performed the task with their left hand,

226 while TMS was delivered over the left motor cortex and MEPs were recorded from a task-

227 irrelevant, right forearm muscle. MEPs were recorded at different times after the Stop signal on

228 different trials: $100-180 \mathrm{~ms}$ in $20 \mathrm{~ms}$ intervals, as well as during the inter-trial interval which

229 served as a baseline. Concurrently, we recorded EMG from the task-relevant left-hand muscles

230 as for studies 1 and 2 above (Fig. 4a). 
The key TMS finding, in keeping with earlier studies ${ }^{25-27}$, was of suppression of MEPs in

232 the task-irrelevant forearm, indicating global motor system suppression, beginning $\sim 140 \mathrm{~ms}$

233 following the Stop signal in Successful Stop trials (Fig. 4b) [see Supplementary Fig. 2 for MEP

234 amplitudes for Partial EMG and No EMG trials separately]. A 2-way repeated measures

235 ANOVA with MEP amplitude as the dependent measure and the factors of trial-type (Correct

236 Go, Successful Stop, Failed Stop) and time (100, 120, 140, 160, $180 \mathrm{~ms}$ after the Stop signal)

237 showed main effects of both trial-type $\left(F(2,32)=7.2, p=0.003, \eta_{p}{ }^{2}=0.3\right)$ and time $\left(F_{G G}(2.5\right.$,

$\left.23840.7)=4.8, p=0.008, \eta_{p}{ }^{2}=0.2\right)$, as well as an interaction of trial-type by time $(F(8,128)=3.4$,

$\left.239 p=0.002, \eta_{p}^{2}=0.2\right)$. Post hoc $t$-tests across Successful Stop and Correct Go trials showed no

240 difference at $100 \mathrm{~ms}\left(t(16)=0.7, p_{\text {Bon }}=1.0, B F_{10}=0.3\right), 120 \mathrm{~ms}\left(t(16)=2.5, p_{\text {Bon }}=0.066, B F_{10}\right.$

$241=2.8)$, and $160 \mathrm{~ms}\left(t(16)=2.1, p_{B o n}=0.159, B F_{10}=1.4\right)$. However, MEP amplitudes were

242 significantly suppressed on Successful Stop trials at $140 \mathrm{~ms}\left(t(16)=4.1, p_{B o n}=0.003, B F_{10}=\right.$

243 39.8) and $180 \mathrm{~ms}\left(t(16)=4.4, p_{B o n}<0.001, B F_{10}=65.2\right)$ after the Stop signal. Therefore, we

244 estimate the onset of the global motor suppression to be $\sim 140 \mathrm{~ms}$ after the Stop signal, which

245 places it $\sim 15 \mathrm{~ms}$ prior to the mean CancelTime (155 $\pm 7 \mathrm{~ms})$. There were no significant

246 differences in MEP amplitudes between Failed Stop and Correct Go trials at any time point,

247 though MEP amplitudes on Successful Stop trials were also suppressed compared to Failed Stop

248 trials at $160 \mathrm{~ms}\left(t(16)=2.9, p_{\text {Bon }}=0.033, B F_{10}=4.9\right)$.

It makes sense that global motor suppression occurs before CancelTime as motor cortical

250 output takes time to be transmitted along the corticospinal pathway to the muscles. To verify

251 whether the $\sim 15 \mathrm{~ms}$ discrepancy in timings could be accounted for by corticospinal conduction

252 delays, we estimated this corticospinal conduction time in a separate phase of the current study

253 by delivering TMS over the hand representation to evoke MEPs in the left, task-relevant, FDI 
254 muscle (Fig. 4c). This was $23 \pm 0.3 \mathrm{~ms}$. Thus, a decline in muscle activity would be expected to

255 be preceded by a reduction in motor cortical output by $\sim 23 \mathrm{~ms}$, which is very similar to the $\sim 15$

256 ms difference between global motor suppression and CancelTime.

257 To further elaborate the temporal relationship between global motor suppression and

258 CancelTime, we performed a trial-by-trial analysis whereby MEP amplitudes were sorted

259 according to the time at which TMS was delivered, relative to the time at which EMG decreased

260 on Successful Stop, Failed Stop and Correct Go trials (Fig. 4d). The suppression of MEPs in

261 Successful Stop trials compared to Correct Go trials began in the $30 \mathrm{~ms}$ prior to the EMG decline

$262 \quad\left(-30\right.$ to $0 \mathrm{~ms}: Z=3.12, p_{\text {Bon }}=0.005 ; 0$ to $30 \mathrm{~ms}: Z=4.48, p_{\text {Bon }}<0.001 ; 30$ to $60 \mathrm{~ms}: Z=2.45$,

$\left.263 p_{B o n}=0.045\right)$. This lag in the time of EMG decrease relative to the time of the MEP suppression

264 on Successful Stop trials can again be accounted for by the corticospinal conduction time. Thus,

265 these results imply that the brain output to task-relevant muscles declines at approximately the

266 same time as the global motor suppression begins. 
a

TMS experimental set-up

EMG recording of MEPs from task-irrelevant ECR muscle
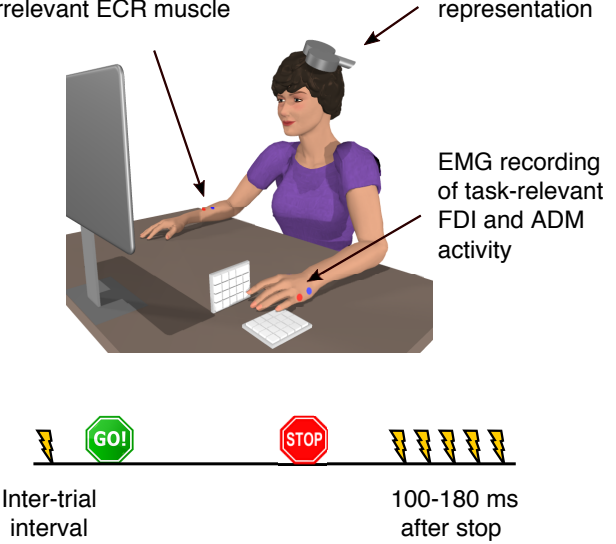

C Estimation of cortispinal conduction time
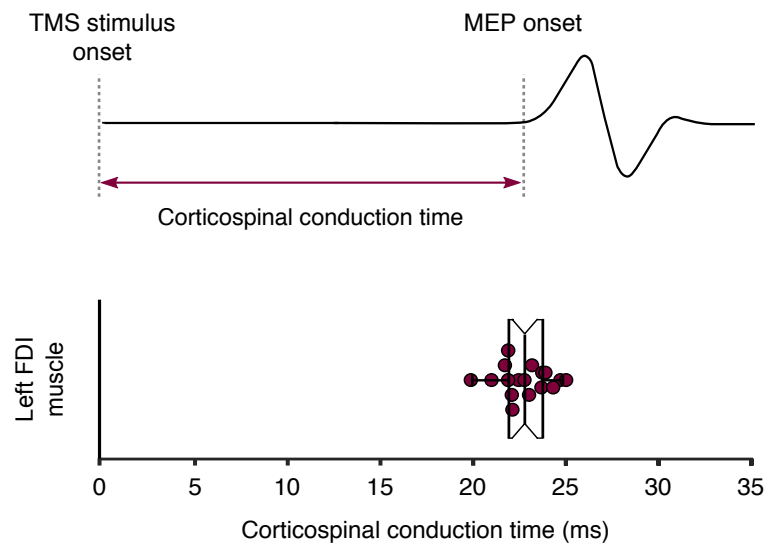

b

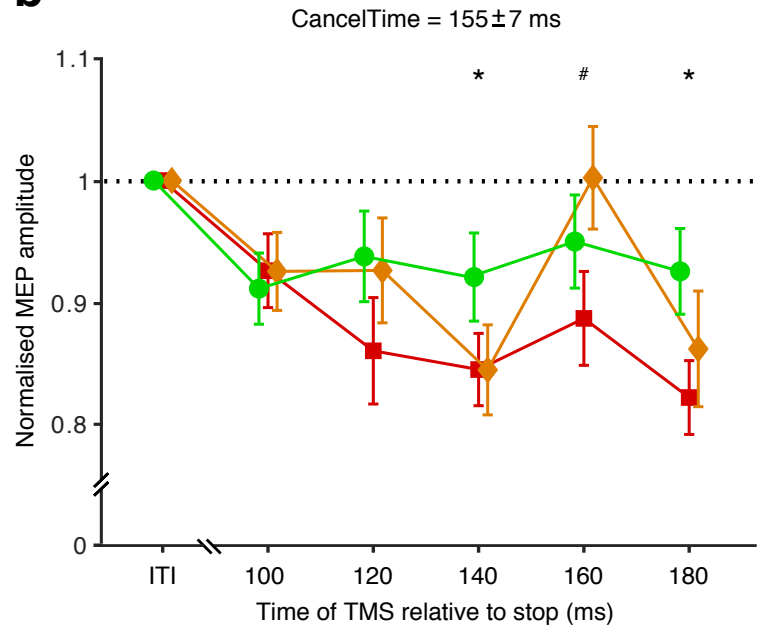

d

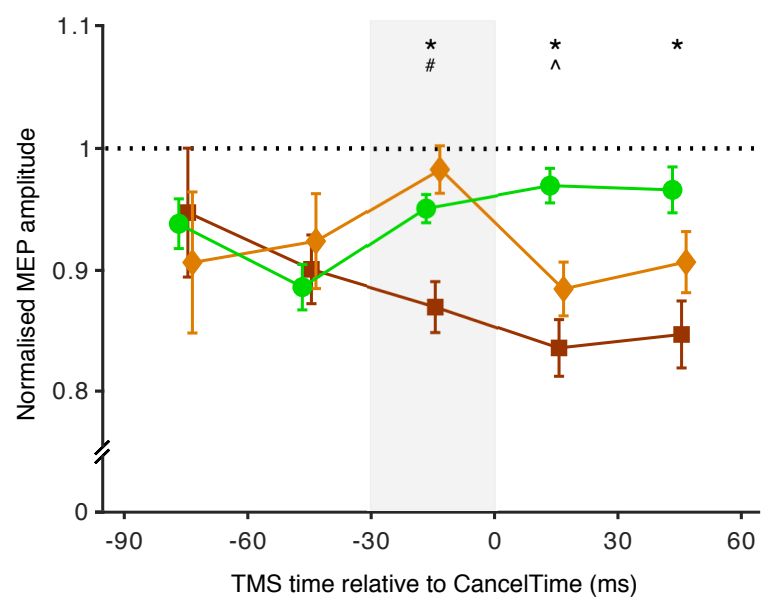

269 Experimental set up and TMS stimulus timings for study 3. Participants performed the Stop signal

270 task with the left hand with concurrent EMG measurement of CancelTime from task-relevant muscles

271 FDI and ADM muscle. On a given trial, a single TMS stimulus over left M1 was delivered at one of 6 possible times to elicit a motor evoked potential (MEP) in the task-irrelevant extensor carpi radialis

(ECR) muscle of the right forearm. (b) Global motor system suppression begins at $140 \mathrm{~ms}$ after the

Stop signal, and thus $\sim 15 \mathrm{~ms}$ prior to the mean CancelTime. Paired $t$-tests: ${ }^{*}, p_{B o n}<0.05$ Successful

Stop (red) vs. Correct Go (green); \#, $p_{B o n}<0.05$ Successful Stop vs. Failed Stop (orange). The black 
dotted line shows amplitude of MEPs normalized to those at the inter-trial interval. (c) (Top) Schematic representation of an MEP. (Bottom) Beeswarm plot of the mean corticospinal conduction time to a hand

277 muscle was established by measuring the onset latency of MEPs in the hand, and was $\sim 23 \mathrm{~ms}$ on average. Each dot represents a participant. This conduction time is included in CancelTime. (d) Trialby-trial analysis of MEP amplitudes organized into $30 \mathrm{~ms}$ time bins reflecting the time of TMS expressed relative to the CancelTime. Global motor system suppression begins in a window 30-0 ms prior to the CancelTime (gray shaded region). Wilcoxon rank sum test: $*, p_{B o n}<0.05$ Partial EMG (brown) vs. Correct Go (green); \#, $p_{B o n}<0.05$ Partial EMG vs. Failed Stop (orange); ${ }^{\wedge}, p_{B o n}<0.05$ Failed Stop vs. Correct Go. The black dotted line shows amplitude of MEPs normalized to those at the inter-trial interval.

285 Study 4 (EEG). Having established that CancelTime reflects the time of an active stopping 286 process at the muscle (Studies 1 and 2, EMG/behavior), which also related tightly with the 287 timing of global motor suppression (Study 3, TMS), we then tested whether this EMG measure 288 was also related to the timing of a prefrontal correlate of action-stopping, specifically the 289 increase of beta power $(13-30 \mathrm{~Hz})$ before $\mathrm{SSRT}_{\mathrm{Beh}}$ at right frontal electrode sites ${ }^{32,33}$. We now 290 measured scalp EEG as well as EMG from the hand, in 11 participants (see Table 1 for 291 behavioral results). We derived beta bursts rather than beta power per se, as bursts have richer 292 features ${ }^{37}$, such as burst timing and duration.

294 used Independent Components Analysis ${ }^{38}\left[\mathrm{see}^{32,33}\right]$. We selected a participant-specific

295 independent component (IC) based on two criteria; First, the scalp topography (right-frontal, and 296 if not present, frontal); and Second, an increase in beta power on Successful Stop trials (from 297 Stop signal to $\mathrm{SSRT}_{\mathrm{Beh}}$; Stop $\mathrm{Win}_{\text {}}$ ) compared to activity prior to the Go cue [-1000 to $-500 \mathrm{~ms}$ 
aligned to the Stop signal; see Methods; Supplementary Fig. 3]. The average scalp topography across all participants is shown in Fig. 5b inset. For each participant, we estimated beta bursts;

First, by filtering the data at the peak beta frequency; and Second, by defining a burst threshold based on the beta amplitude in a baseline period after the Stop signal (500-1000 ms after Stop signal in the Stop trials, and 500-1000 ms after the mean SSD in the Correct Go trials) (see Methods; Supplementary Fig 4). Failed Stop and Correct Go trials prior to $\operatorname{SSRT}_{\mathrm{Beh}}$ (Fig. 5a). To quantify this across participants, we compared the mean burst $\%$ among the 3 trial-types, and for the time window from the Stop signal to the $\mathrm{SSRT}_{\mathrm{Beh}}$ of a participant (Stop ${ }_{\mathrm{Win}}$ ) and the baseline period before the Stop signal

308 (Base ${ }_{W i n}$; Go to Stop signal in Stop trials and Go to mean SSD in Correct Go trials). We performed a 2-way repeated measures ANOVA with mean burst \% as the dependent measure,

310 with trial-type (Successful, Failed Stop, and Correct Go trials) and time-window (Stop win and

311 Base $\left._{\mathrm{Win}}\right)$ as factors. There was a significant main effect of trial-type $(F(2,20)=4.5, p=0.025$,

$\left.312 \eta_{p}{ }^{2}=0.3\right)$ and a trial-type by time-window interaction $\left(F(2,20)=4.0, p=0.034 \eta_{p}{ }^{2}=0.3\right)$, but no

313 main effect of time-window $\left(F(1,10)=3.8, p=0.088, \eta_{p}{ }^{2}=0.3\right)$. Post hoc $t$-tests showed that in

314 the Stop ${ }_{W i n}$ there was a significant increase in burst \% for Successful Stop $(14.6 \pm 1.7 \%)$

315 compared to both its baseline $\left(9.9 \pm 1.7 \% ; t(10)=3.3, p_{B o n}=0.022, B F_{10}=7.6\right)$, and Correct Go

$316\left(9.6 \pm 1.3 \% ; t(10)=3.7, p_{B o n}=0.015, B F_{10}=11.8\right)$, but not to Failed Stop $(10.3 \pm 1.6 \% ; t(10)=$

$\left.3172.1, p_{B o n}=0.198, B F_{10}=1.2\right)($ Fig. $5 b)$. Thus, burst \% increased for the Successful Stop trials. To further clarify the temporal relationship between beta activity and our EMG measure

319 of action-stopping, we quantified the mean burst time (BurstTime in the Stop win) for each

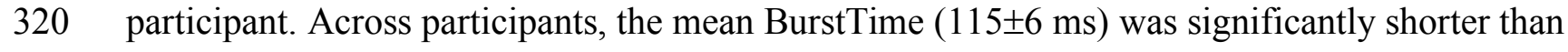


321 mean CancelTime $\left(169 \pm 10 \mathrm{~ms} ; t(10)=8.2, p<0.001, B F_{10}>100\right)$ and there was also a strong

322 positive relationship between them $\left(\rho=0.76, p=0.006, B F_{10}=10.6\right.$; Fig. $\left.5 \mathrm{~d}\right)$ [see

323 Supplementary Fig. 5 for correlation between CancelTime and other burst parameters]. Further,

324 we show that the observed correlation was not merely an artifact of varying Stop win across

325 participants (permutation test, $p<0.05$; see Methods). Thus, these results show that participants

326 with an early frontal beta burst also had an early CancelTime.

a

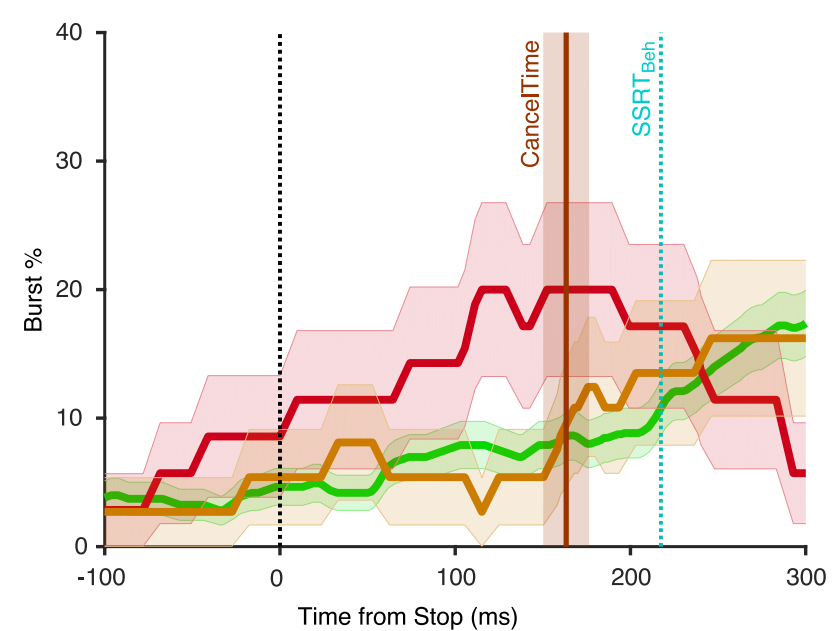

Time from Stop $(\mathrm{ms})$

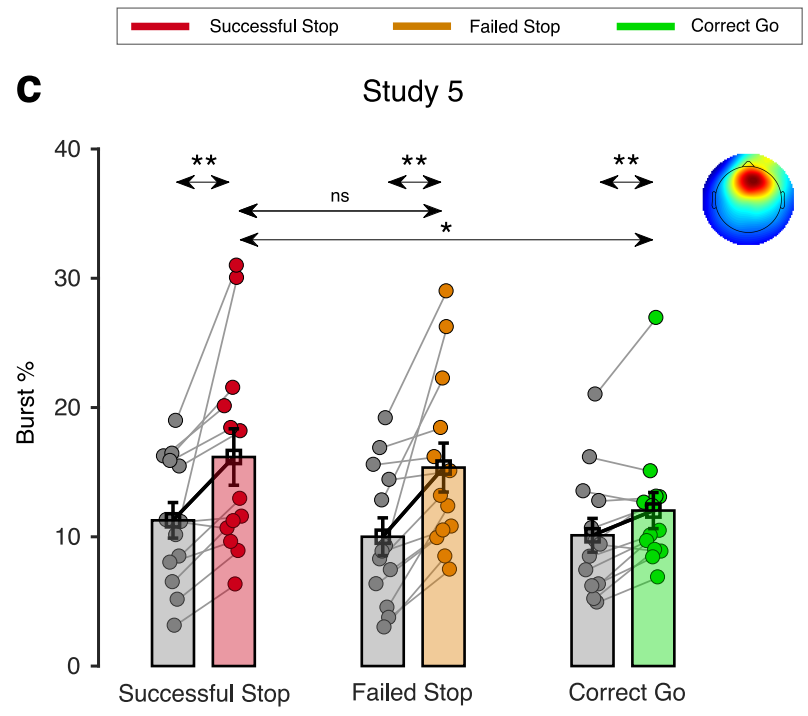

b

Study 4

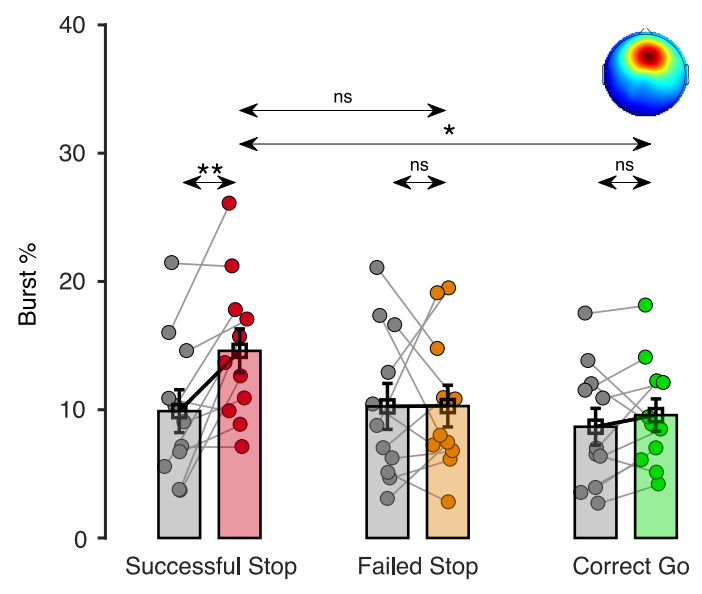

d

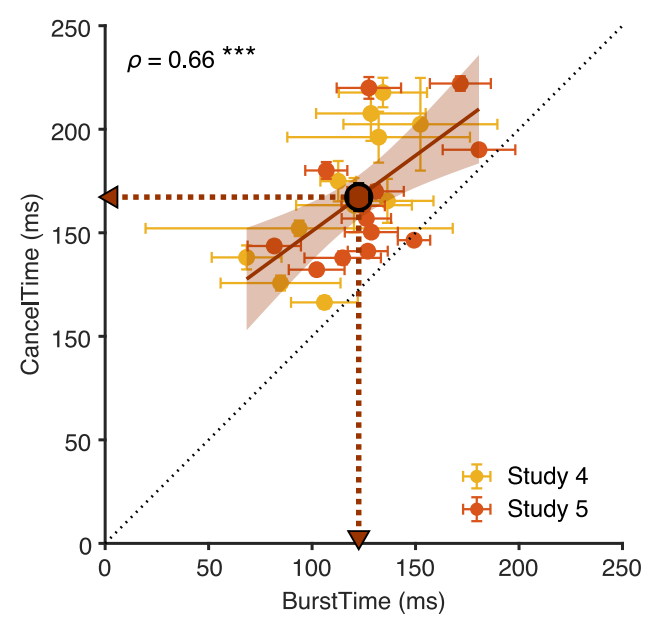


Figure 5 | Relationship between scalp EEG beta bursts and CancelTime (study 4 and 5). (a) Burst

$\%$ across time for Successful Stop (red), Failed Stop (orange), and Correct Go (green) trials for an

exemplar participant in study 4 from the right frontal spatial filter. The shaded region represents

mean \pm s.e.m. The CancelTime is shown in brown and the $\mathrm{SSRT}_{\mathrm{Beh}}$ as a cyan line. (b) The mean burst

334 probability across all participants for Successful Stop (red), Failed Stop (orange), and Correct Go

335 (green) trials and their respective baselines (gray). The bars and cross-hairs represent the mean and s.e.m across participants, while the dots represent individual participants. (Inset top right) The average scalp topography of all the right frontal ICs across all participants. (c) Same as (b) but for study 5. (d) Correlation between mean BurstTime and mean CancelTime. The yellow dots and crosshairs represent the participants in study 4 , while the light red ones represent participants in study 5 . The brown line and the shaded area represent the linear regression fit and its $95 \%$ confidence interval (pooled study 4 and 5). Other details same as Fig. 2 d.

342 Study 5 (EEG replication): We ran a new sample of 13 participants (see Table 1 for behavioral 343 results). As above a right frontal IC was extracted for each participant (average topography Fig.

3445 c inset, see Supplementary Fig 3) and the burst \% was compared for the 3 trial-types

345 (Successful Stop, Failed Stop, and Correct Go) in the two time-windows (Stopwin and Base Win $_{\text {). }}$

346 Again, a 2-way repeated measures ANOVA with burst \% as the dependent measure revealed that

347 there was a significant main effect of trial-type $\left(F(2,24)=6.9, p=0.004, \eta_{p}{ }^{2}=0.4\right)$ and a trial-

348 type by time-window interaction $\left(F(1,12)=5.8, p=0.009, \eta_{p}{ }^{2}=0.3\right.$; Fig. $\left.5 \mathrm{c}\right)$. Here there was

349 also a significant effect of time-window on burst $\%\left(F(1,12)=16.1, p=0.002, \eta_{p}{ }^{2}=0.6\right)$. Post-

350 hoc $t$-tests confirmed that the burst \% was greater for Successful Stop (16.2 $2.2 \%)$ compared to

351 its baseline $\left(11.3 \pm 1.4 \% ; t(12)=3.3, p_{B o n}=0.021, B F_{10}=7.6\right)$, and Correct Go (12.0 $\pm 1.4 \%$;

$\left.352 t(12)=3.0, p_{\text {Bon }}=0.030, B F_{10}=5.3\right)$ but not compared to Failed Stop $(15.4 \pm 1.4 \% ; t(12)=1.0$, 
$\left.353 p_{B o n}=0.957, B F_{10}=0.34\right)$. Across participants, the mean BurstTime $(129 \pm 7 \mathrm{~ms})$ was again

354 significantly shorter than CancelTime $\left(166 \pm 8 \mathrm{~ms} ; t(10)=5.0, p<0.001, B F_{10}>100\right)$ and there

355 was a significant positive relationship $\left(\rho=0.57, p=0.045, B F_{10}=1.9\right.$; Fig. $\left.5 \mathrm{~d}\right)$. Again, a

356 permutation test suggested that this correlation was unlikely to result from mere variation in the

357 length of Stop ${ }_{W i n}$ across participants $(p<0.05)$. Combining data from studies 4 and 5 confirms

358 the strong relationship between right frontal beta BurstTime and CancelTime $(\rho=0.66, p<$

$\left.3590.001, B F_{10}=29.4\right)$.

361 Discussion

This set of studies provides detailed information about the timing of subprocesses in

363 human action-stopping. We started with the recently published observations that the standard

364 behavioral measure of action-stopping (SSRT) is, an over-estimate of stopping speed ${ }^{15,21,22}$. To

365 more precisely delve into this, we developed and validated a trial-by-trial method for estimating

366 stopping speed from EMG. We focused on Successful Stop trials with small impulses (partial

367 bursts) in EMG activity. The amplitude of such partial EMG activity was $~ 50 \%$ of the amplitude

368 of EMG activity for outright keypresses, and this decreased at $\sim 160 \mathrm{~ms}$ after the Stop signal

369 (CancelTime). While, one interpretation of this partial EMG activity is that it merely reflects

370 'weak' Go activation that did not run to completion, several lines of evidence strongly suggest it

371 is a muscle manifestation of the stopped response. First, CancelTime had a strong positive

372 correlation with $\mathrm{SSRT}_{\mathrm{Beh}}$. Second, the variability of CancelTime was positively correlated with

373 the variability of SSRT estimated from the BEESTS modeling framework. Third, the partial

374 EMG activity had a profile which was initially similar to the EMG profile seen when actual

375 keypresses were made, and only diverged at $~ 55 \mathrm{~ms}$ after EMG onset. This initial similarity 
376 would not be expected if it were a weak Go activation - since previous research has

377 demonstrated that weak and strong muscle activations have distinct profiles that diverge soon

378 after onset ${ }^{39}$. Fourth, our TMS experiment demonstrated that, CancelTime coincided well with

379 the timing of a putative basal ganglia-mediated global motor suppression ${ }^{25-30}$. This implies that

380 the smaller amplitude and earlier decline of the partial EMG activity on Successful Stop was due

381 to an active suppression of motor output. Fifth, across participants, on Successful Stop trials,

382 CancelTime correlated strongly with the time of right frontal beta bursts (BurstTime) from scalp

383 EEG. This is consistent with response inhibition being implemented via right prefrontal cortex ${ }^{12}$,

384 and with previous research showing an increase of beta at right frontal electrode sites before

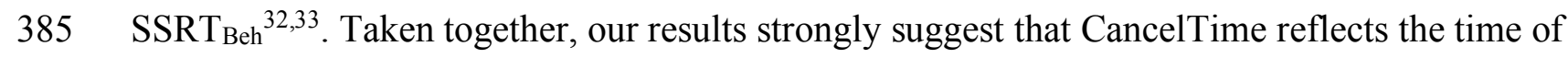

386 implementation of an active Stop process at the muscle-level. These results have striking

387 theoretical and practical implications for response inhibition research and, more widely, our

388 understanding of impulse control.

Notably, CancelTime was $\sim 60 \mathrm{~ms}$ earlier than $\mathrm{SSRT}_{\text {Beh. }}$. To better understand this

390 discrepancy, we calculated SSRT based on the EMG response rather than behavior. We saw that

391 SSRT $_{\text {EMG }}$ better matched CancelTime than did SSRT Beh. Thus, $_{\text {SSRT }}$ Beh could be an over-

392 estimation of the duration of the Stop process in the brain. This extra time in SSRT $\mathrm{Beh}_{\text {probably }}$

393 reflects a 'ballistic stage' in generation of the button press ${ }^{40,41}$. We suggest that the maximum

394 CancelTime reflects the last point at which a Stop process can intervene to prevent responses.

395 We note that CancelTime (a muscle measurement) is an overestimation of the brain's stopping

396 speed since it does not include the corticospinal conduction time, which we estimated at $\sim 20 \mathrm{~ms}$.

397 Indeed, our TMS results show that global motor suppression, which we take as the time at which

398 motor areas of the brain are suppressed, is $\sim 140 \mathrm{~ms}$ (which is $\sim 15 \mathrm{~ms}$ less than CancelTime). 
One important consequence of our observation that the brain's stopping speed is $\sim 140 \mathrm{~ms}$ is that

400 neural events that mediate stopping need to occur before this time. Indeed, we found that right

401 frontal beta activity increased $\sim 120 \mathrm{~ms}$ after the Stop signal on Successful Stop trials, and also

402 that, across participants, there was a strong positive relationship between mean BurstTime and mean CancelTime.

Taken together, these studies motivate a detailed model of the temporal events of action-

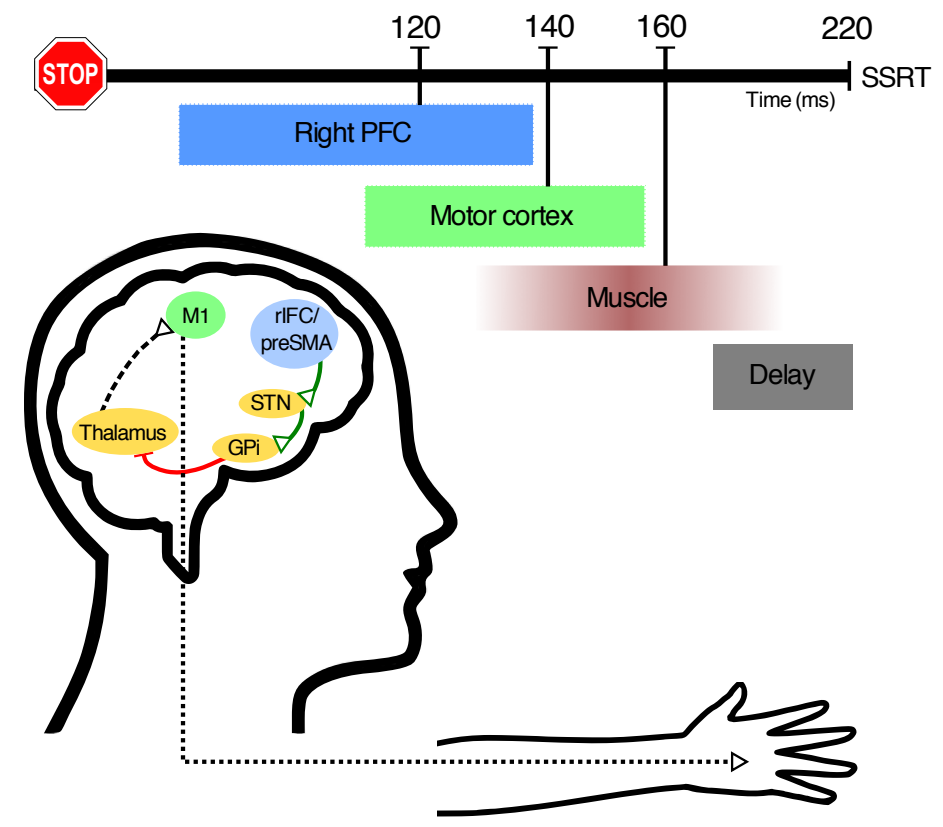


Figure 6 | Hypothetical model of the temporal cascade of processes underlying human action-

stopping. Following the Stop signal, the right PFC including the rIFC and the preSMA gets activated at $\sim 120 \mathrm{~ms}$. These region/s activate the STN of the basal ganglia which in turn activates the globus pallidus interna which via its inhibition on the motor regions of the thalamus cuts down the 'drive' of

419 suppression is reflected in the hand muscle at $\sim 160 \mathrm{~ms}$ which is measured as the CancelTime. There is a delay of $\sim 60 \mathrm{~ms}$ at the muscle level which gets added to the behavioral estimate of SSRT.

This model specifies the chronometrics of stopping in more detail than extant human

423 models, and, more generally, raises questions about the timing reported in some other studies.

424 For example, other research has shown that movement neurons in monkey Frontal Eye Field

425 decrease activity $<10 \mathrm{~ms}$ before $\mathrm{SSRT}^{42}$, dopaminergic neurons in rodent substantia nigra and

426 striatum increase activity $12 \mathrm{~ms}$ prior to $\mathrm{SSRT}^{43}$, TMS at $\sim 25 \mathrm{~ms}$ before SSRT over human

427 Intraparietal Sulcus prolongs SSRT ${ }^{44}$, and that P300 human EEG activity 300 ms after the Stop

428 signal relates to stopping speed ${ }^{45}$. Our observations of shorter latencies for prefrontal bursts,

429 TMS-MEP and muscle CancelTime, raise questions about what is reflected in these late neural

430 activities.

431 Our results have several important implications. First, as just noted, they provide

432 temporal constraints on neuroscience studies of stopping in the brain. They suggest that methods

433 with high temporal resolution need to focus on the time after the Stop signal and before

434 CancelTime (indeed CancelTime minus conduction time) rather than before $\mathrm{SSRT}_{\mathrm{Beh}}$, and we

435 provide a novel single-trial metric of stopping. Second, our results have clinical implications.

436 Whereas meta-analysis shows that $\mathrm{SSRT}_{\mathrm{Beh}}$ is longer for patients (e.g. ADHD, OCD, and 
437 substance use disorder) vs. controls $^{6-11}$, not all such studies show differences ${ }^{8,46-48}$. We predict

438 that our new single-trial method of CancelTime will be more sensitive than SSRT $_{\text {Beh. }}$

439 Furthermore, future studies can easily estimate within-subject variability in CancelTime, which

440 will likely discriminate patients from controls. Third, our results provide insight into why

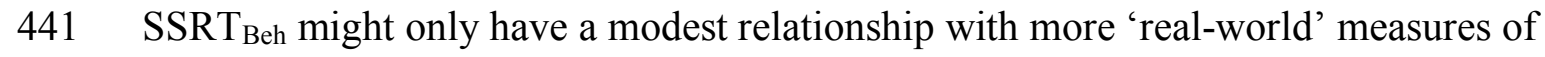

442 impulsivity ${ }^{15-20}$. As we show, the SSRT $_{\text {Beh }}$ includes not only CancelTime but an extra, and

443 variable, $60 \mathrm{~ms}$ ballistic stage. We expect that future studies may show stronger correlations

444 between CancelTime and self-report than that seen between $\mathrm{SSRT}_{\mathrm{Beh}}$ and self-report (also see ${ }^{15}$ );

445 likewise we predict that right frontal beta burst time might also correlate more tightly with self-

446 report measures. More generally, the detailed timing information of frontal beta at $\sim 120 \mathrm{ms,}$

447 global motor suppression at $\sim 140 \mathrm{~ms}$, and CancelTime at $\sim 160 \mathrm{~ms}$ points to subprocesses of

448 action-stopping that provide potential biomarkers that could better explain individual differences

449 in impulse control.

450 In conclusion, we provide a detailed timing model of action-stopping that partitions it

451 into subprocesses that are isolable to different nodes and are surely more precise than the

452 behavioral speed of stopping. At the core of this timing model is a novel method of measuring

453 the speed of stopping from the muscles. This provides a single-trial estimate of stopping speed

454 that could be easily measured with minimal equipment in any lab that studies human

455 participants.

456

457 Methods 
458 Participants. All were adult, healthy, human volunteers who provided written informed consent

459 and were compensated at $\$ 20 /$ hour. The studies were approved by the UCSD Institutional

460 Review Board.

Study 1. Ten participants (4 females; age 22 11 years; all right-handed).

464 Failed Stop RT < Correct Go RT, and P(Stop) increasing monotonically as a function of SSD), 465 and two were excluded for noisy EMG data. left-handed) with no contraindications to $\mathrm{TMS}^{49}$. One was excluded for bad behavior.

$$
\text { Study } 4 \text { (EEG). Eleven participants (6 females, age } 19 \pm 0.4 \text { years, all right-handed) }
$$

469 participated.

Study 5 (EEG): Fifteen participants (9 females, age 21 \pm 0.4 years, all right-handed)

471 participated. Two were excluded from analysis, one for misaligned EEG markers due to a

472 technical issue, while the other lacked a right frontal brain IC, based on our standard method ${ }^{32,33}$.

474 Stop-signal task. This was run with MATLAB 2014b (Mathworks, USA) and Psychtoolbox ${ }^{50}$.

475 Each trial began with a white square appearing at the center of the screen for $500 \pm 50 \mathrm{~ms}$. Then a

476 right or left white arrow appeared at the center. When the left arrow appeared, participants had to 477 press a key on a vertically oriented keypad using their index finger, while for a right arrow they 478 had to press down on a key on a horizontally oriented keypad with their pinky finger (Fig. 1b 479 inset), as fast and as accurately as possible (Go trials). The stimuli remained on the screen for 1 480 s. If participants did not respond within this time, the trial aborted, and 'Too Slow' was 
481 presented. On $25 \%$ of the trials, the arrow turned red after a stop signal delay (SSD), and

482 participants tried to stop the response (Stop trials). The SSD was adjusted using two independent

483 staircases (for right and left directions), where the SSD increased and decreased by $50 \mathrm{~ms}$

484 following a Successful Stop and Failed Stop respectively. Each trial was followed by an inter-

485 trial interval (ITI) and the entire duration of each trial including the ITI is $2.5 \mathrm{~s}$ (Fig. 1a).

486 Study 1 and 2. Participants performed the task with their right hand. They performed 40 practice

487 trials before the actual experiment, where their baseline SSD was determined and was

488 subsequently used as the starting SSD in the main experiment. In study 1 and 2, the experiment

489 had 600 trials divided in 15 blocks, such that each block had 40 trials (450 Go trial and 150 Stop

490 trials). At the end of each block the participants were presented a figure showing their mean

491 reaction times (RT) in each block. Participants were verbally encouraged to maintain their mean

492 reaction time constant across the different blocks and between $0.4-0.6 \mathrm{~s}$.

493 Study 3. Participants performed the task with their left hand. Following 48 practice trials without

494 TMS, participants performed 12 blocks of the experiment with TMS, with each block consisting

495 of 96 trials each (72 Go trials and 24 Stop trials).

496 Study 4. Participants performed the task with their right hand. Following 160 practice trials,

497 participants performed 4 blocks of 80 trials (240 Go trials and 80 Stop trials).

498 Study 5. Participants performed the task with their right hand. Following 80 practice trials,

499 participants 24 blocks of 80 trials each (1440 Go trials and 480 Stop trials).

501 EMG recording. EMG data were acquired using a Grass QP511 AC amplifier (Glass

502 Technologies, West Warwick, RI) with a frequency cut-off between 30 and $1000 \mathrm{~Hz}$. A CED

503 Micro $1401 \mathrm{mk}$ II acquisition system sampled the data at $2 \mathrm{kHz}$. The EMG data were acquired by 
504 CED Signal v4 software (Cambridge Electronic Design Limited, Cambridge, UK) for $2 \mathrm{~s}$

505 following the fixation cue. The data acquisition was triggered from MATLAB using a USB-

506 1208FS DAQ card (Measuring Computing, Norton, MA). In all 5 experiments, surface EMG

507 was recorded from both the first dorsal interossei (FDI) and the abductor digiti minimi (ADM)

508 muscles of the hand (Fig. 1b inset). In the TMS experiment, surface EMG was also recorded

509 from the task-irrelevant right extensor carpi radialis (ECR) muscle (Fig. 5a).

511 TMS. MEPs were evoked using a TMS device (PowerMag Lab 100, MAG\&More GMBH,

512 Munich, Germany) delivering full sine wave pulses, and connected to a figure-of-eight coil (70

513 mm diameter, Double coil PMD70-pCool; MAG\&More GMBH, Munich, Germany). During the

514 task, the coil was positioned on the scalp over the left primary motor cortex representation of the

515 ECR muscle and oriented so that the coil handle was approximately perpendicular to the central

516 sulcus, i.e. at $\sim 45^{\circ}$ to the mid-sagittal line, and the initial phase of current induced in the brain

517 was posterior-to-anterior across the central sulcus. Prior to the experiment, the motor hot spot

518 was determined as the position on the scalp where slightly supra-threshold stimuli produced the

519 largest and most consistent MEPs in ECR. The position was marked on a cap worn by the

520 participants. Resting motor threshold (RMT) was defined as the lowest intensity to evoke an

521 MEP of at least $0.05 \mathrm{mV}$ in 5 of 10 consecutive trials while participants were at rest. We then

522 established the test stimulus intensity to be used during task, which was set to produce a mean

523 MEP amplitude of approximately $0.2-0.5 \mathrm{mV}$ whilst the participant was at rest.

MEPs were also evoked in the left FDI muscle prior to beginning the main experiment

525 for the purpose of recording the corticospinal conduction time. The motor hot spot for the FDI

526 was defined in a manner similar to that for the ECR. The active motor threshold (AMT) was 
527 defined as the lowest intensity to evoke a discernible MEP in 5 of 10 consecutive trials, while

528 participants maintained slight voluntary contraction $(\sim 10 \%$ of maximum voluntary EMG

529 amplitude during isometric finger abduction). Then, 10 stimuli were delivered at 150\% AMT

530 during slight voluntary contraction (again 10\% of maximum), with the coil oriented to induce

531 lateral-medial current in the brain in order to obtain estimates of corticospinal conduction time.

532 During the task, TMS stimuli were delivered on every Stop trial and on 50\% of Go trials.

533 On every Stop trial, a single TMS stimulus at the test stimulus intensity was delivered at one of

534 six time points: inter-trial interval (100 ms prior to fixation; ITI), $100 \mathrm{~ms}, 120 \mathrm{~ms}, 140 \mathrm{~ms}, 160$

$535 \mathrm{~ms}$ and $180 \mathrm{~ms}$ after the Stop signal (Fig. 4a). On the Go trials, TMS stimuli were yoked to the

536 time of the Stop signal on the previous Stop trial. Thus, there were 48 trials per TMS time point

537 on Stop trials and 96 trials per time point on Go trials.

539 EEG. 64 channel EEG (Easycap, Brainvision LLC) was recorded in the standard 10/20

540 configuration at $1 \mathrm{kHz}$.

542 Data analysis. All analyses were performed using MATLAB (R2016b, R2018b, R2019a).

543 Stop Signal Reaction Time. SSRT from the behavioral responses (SSRT $\mathrm{Beh}$ ) was determined

544 using the integration method ${ }^{5}$. When calculating SSRT using the EMG responses, $\mathrm{SSRT}_{\mathrm{EMG}}$, as

545 the $\mathrm{P}$ (Respond|Stop) was often much more than 0.5, we calculated the SSRT individually for the

5463 most frequent SSDs and then averaged it ${ }^{51}$.

547 EMG data analysis. EMG data were filtered using $4^{\text {th }}$ order Butterworth filter (roll-off 24

$548 \mathrm{~dB}$ /octave) to remove $60 \mathrm{~Hz}$ noise and its harmonics at 120, and $180 \mathrm{~Hz}$. EMG data were full-

549 wave rectified and the root-mean square (RMS) of the signal was computed using a centered 
550 window of $50 \mathrm{~ms}$. Any EMG activity which was greater than 8 SD of the mean EMG activity in

551 the baseline period (Fixation to Go cue) was marked, on a trial-by-trial basis. Starting from the

552 peak of that EMG activity, the onset was marked at the point where the activity dropped below

$55320 \%$ of the peak for 5 consecutive ms. This method of adjusting the threshold based on the peak

554 EMG activity, allowed better onset detection than a fixed threshold, especially when the

555 amplitude of the EMG activity was small. The time when EMG started to decline was

556 determined as the time when, following the peak EMG activity, the activity decreased for 5

557 consecutive ms. Visual inspection of individual trials showed that this method provided a reliable

558 detection of both EMG onsets (see Supplementary Fig. 1a, 1b for EMG onset vs. RT correlation)

559 and decline. Any detected EMG timing which was beyond 1.5 times the inter-quartile range

560 (IQR) of the first and third quartile (Q3) of that particular timing distribution was deemed an

561 outlier. This removed $<4 \%$ trials. CancelTime was marked as the time of the decline following

562 the Stop signal. For outlier rejection, CancelTimes had a lower cutoff of $50 \mathrm{~ms}$ and higher cutoff

563 of Q3+1.5×IQR. This removed $<3 \%$ trials.

564 As the peak EMG amplitude for the FDI and ADM muscle were quite distinct, before

565 averaging the two EMG activities, we normalized the muscle activity by the peak activity in that

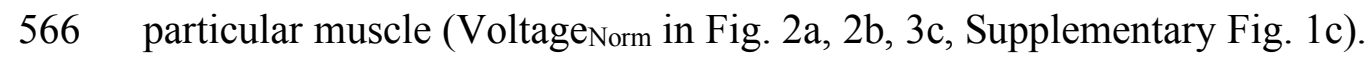

567 Global MEP suppression. MEP amplitudes were measured on a trial-by-trial basis. Data were

568 included for analysis if the following criteria were met: (i) the amplitude of the ECR EMG signal

569 in a $90 \mathrm{~ms}$ period prior to the TMS stimulus was $<0.05 \mathrm{mV}$; (ii) the amplitude of the MEP fell

570 within the mean $\pm 1.5 \times \mathrm{IQR}$ of values for the same time point and trial type (Correct Go, Failed

571 Stop, Successful Stop). Thereafter, MEP amplitudes measured at the ITI were collapsed across

572 trial type (Correct Go, Failed Stop and Successful Stop), averaged and used as a baseline against 
573 which to compare other TMS time points. For each of the other TMS time points $(100,120,140$,

$574160,180 \mathrm{~ms}$ following the Stop signal), data were averaged within each trial type (Correct Go,

575 Failed stop, Successful Stop) and expressed as a percentage of the mean ITI MEP amplitude.

576 Corticospinal conduction time. Corticospinal conduction time was determined by delivering

577 TMS over the hand representation of left FDI and measuring MEP from the muscle (Fig. 4c).

578 The earliest MEP onset latency across 10 trials was identified by visual inspection of the EMG

$579 \operatorname{traces}^{52-54}$.

580 Trial-by-trial analysis of the time of the CancelTime and time of global motor suppression. To

581 compare the temporal association between the EMG decline and MEP suppression, we

582 performed a trial-by-trial analysis of stop-signal task data only on trials where an EMG burst was

583 detected. We first normalized the time of TMS on a given trial by subtracting the time of EMG

584 decline from the time of the TMS pulse. Hence, negative values mean that TMS was delivered

585 before the EMG decline and positive values mean that TMS was delivered after. We then plotted

586 MEP amplitudes for each of the three response types (Correct Go, Failed Stop, and Successful

587 Stop) against the normalized times binned into $30 \mathrm{~ms}$ windows. This analysis meant that for a

588 given individual there were relatively few trials per time bin, and some bins would occasionally

589 contain no data. Therefore, we combined data across all individuals. Prior to this, MEP

590 amplitudes for each individual were normalized to the mean MEP amplitude at the inter-trial

591 interval, to account for inter-individual variability in absolute MEP amplitudes at baseline. We

592 restricted our analysis to time bins that contained at least 50 trials, which resulted in time range -

$59390 \mathrm{~ms}$ to $60 \mathrm{~ms}$.

594 EEG Preprocessing. We used EEGLAB ${ }^{55}$ and custom-made scripts to analyze the data. The data

595 were downsampled to $512 \mathrm{~Hz}$ and band-pass filtered between 2-100 Hz. A $60 \mathrm{~Hz}$ and $180 \mathrm{~Hz}$ 
596 FIR notch filter were applied to remove line noise and its harmonics. EEG data were then re-

597 referenced to the average. The continuous data were visually inspected to remove bad channels

598 and noisy stretches.

599 ICA analysis. The noise-rejected data were then subjected to logistic Infomax ICA to isolate

600 independent components (ICs) for each participant separately ${ }^{38}$. We then computed the best-

601 fitting single equivalent dipole matched to the scalp projection for each IC using the DIPFIT

602 toolbox in EEGLAB ${ }^{55,56}$. ICs representing non-brain activity related to eye movements, muscle,

603 and other sources were first identified using the frequency spectrum (increased power at high

604 frequencies), scalp maps (activity outside the brain) and the residual variance of the dipole

605 (greater than 15\%) and then, subtracted from the data. A putative right frontal IC was then

606 identified from the scalp maps (if not present then we used frontal topography) and the channel

607 data were projected onto the corresponding right frontal IC. The data on Successful Stop trials

608 were then epoched from $-1.5 \mathrm{~s}$ to $1.5 \mathrm{~s}$ aligned to the Stop signal. We estimated the time-

609 frequency maps from 4 to $30 \mathrm{~Hz}$, and -100 to $400 \mathrm{~ms}$ using Morlet wavelets with 3 cycles at low

610 frequencies linearly increasing by 0.5 at higher frequencies. The IC was selected only if there

611 was a beta power $(13$ to $30 \mathrm{~Hz})$ increase in the window between the Stop signal and SSRT $\mathrm{Beh}$

612 compared to a time-window prior to the Go cue (-1000 to $-500 \mathrm{~ms}$ aligned to Stop signal). In

613 each participant, the beta frequency which had the maximum power in this time window was

614 used in the beta bursts computation (Supplementary Fig. 3).

615 Beta Bursts. To estimate the beta bursts, the epoched data were first filtered at the peak beta

616 frequency using a frequency domain Gaussian window with full-width half-maximum of $5 \mathrm{~Hz}$.

617 The complex analytic envelope was then obtained by Hilbert transform, and its absolute value

618 provided the power estimate. In each participant, to define the burst threshold, the beta amplitude 
619 within a period of 500 to $1000 \mathrm{~ms}$ (i.e. after the Stop signal in the Stop trials, and after the mean

620 SSD in the Correct Go trials) was pooled across all trials [compared to the ICA analysis here we

621 picked a different time-window to estimate the burst threshold to keep the analysis unbiased.

622 However, picking the same time-window also yielded similar results]. The threshold was set as

623 the median + 1.5 SD of the beta amplitude distribution (Supplementary Fig. 4). Once the burst

624 was detected, the burst width threshold was set as the median +1 SD. We binary-coded each

625 time point where the beta amplitude crossed the burst width threshold to compute the burst \%

626 across trials. For each detected burst, the time of the peak beta amplitude was marked as the

627 BurstTime.

629 Statistical analysis. For pairwise comparisons, the data were first checked for normality using

630 Lilliefors test, and if normally distributed a two-tailed $t$-test ( $t$-statistic) was performed, else a

631 Wilcoxon signed rank test (Z-statistic) was performed. We interpret the effect sizes as small

632 (Cohen's $d$ : 0.2-0.5; Bayes Factor in favor of the alternate hypothesis, $\left.B F_{10}: 1-3\right)$, medium $(d$ :

$\left.6330.5-0.8 ; B F_{10}: 3-10\right)$, large $\left(d>0.8 ; B F_{10}>10\right)$. For comparisons across multiple levels, repeated-

634 measures ANOVA was used, followed by Bonferroni corrected $t$-tests for pairwise comparisons

635 (Bonferroni corrected $p$-value: $p_{B o n}$ ). The Greenhouse-Geisser correction was applied where the

636 assumption of sphericity in ANOVA was violated (corrected $F$-statistic: $F_{G G}$ ). Effect sizes for

637 ANOVAs were interpreted as small (partial eta-squared, $\left.\eta_{p}^{2}: 0.01-0.06\right)$, medium $\left(\eta_{p}^{2}: 0.06-\right.$

$6380.14)$, and large $\left(\eta_{p}^{2}>0.14\right)$. For correlational analyses, Pearson's correlation coefficient $(r)$ was

639 usually used, but Spearman's correlation coefficient $(\rho)$ was used when the data bounded in a

640 closed interval. All data are presented as mean \pm s.e.m. 
In testing the relationship between BurstTime and CancelTime, we performed a

permutation test. We sampled BurstTimes randomly from a uniform distribution between 0 and

643 SSRT $_{\text {Beh }}$ for a given participant for 3000 iterations. For each iteration, we then computed the

644 correlation $(r)$ between the mean BurstTime and the mean CancelTime across participants. This

645 generated a distribution of $r$ ranging between -1 and 1. The $p$-value for our analysis was

646 determined as the $\mathrm{P}\left(r \geq r_{O b s} \mid \mathrm{H}_{0}\right)$ in the permuted data.

\section{Bayesian modelling of behavioral data}

We used the BEESTs model developed by Dora Matzke and colleagues (run in R Studio

650 1.1.463) which assumes a race between two stochastically independent process, a Go and a Stop

651 processes. This model estimates the distribution of the SSRT by using the participant's Go RT

652 distribution, and by considering the Failed Stop RTs as a censored Go RT distribution. The

653 censoring points are sampled randomly from the SSRT distribution on each Stop trial. The RT

654 distributions underlying the Go and Stop process is assumed to have a Gaussian and an

655 exponential component and is described 3 parameters $\left(\mu_{G o}, \sigma_{G o}, \tau_{G o}\right.$ and $\left.\mu_{\text {Stop }}, \sigma_{\text {Stop }} \tau_{\text {Stop }}\right)$. For

656 such ex-Gaussian distributions, the mean and variance of the RT distribution are determined as $\mu$

$657+\tau$ and $\mu^{2}+\tau^{2}$, respectively. The model also estimates the probability of trigger failures for each

658 participant. The model uses Bayesian Parametric Method (BPE) to estimate the parameters of the

659 distributions. We used a hierarchical BPE, where individual subject parameters are modeled with

660 the group-level distributions. This approach is thought to be more accurate than fitting individual

661 participants and is effective when there is less data per participant ${ }^{57}$. We pooled the subjects

662 across both study 1 and 2 to estimate the individual parameters. The priors were bounded

663 uniform distributions $\left(\mu_{G o}, \mu_{\text {Stop }}: U(0,2) ; \sigma_{G o}, \sigma_{\text {Stop }}: U(0,0.5) \tau_{G o,} \tau_{\text {Stop }}: U(0,0.5) ; \mathrm{pTF}: \mathrm{U}(0,1)\right)$. 
664 The posterior distributions were estimated using the Metropolis-within-Gibbs sampling and we

665 ran multiple chains. We ran the model for 5000 samples with a thinning of 5. The Gelman-Rubin

$666(\hat{\mathrm{R}})$ statistic was used to estimate the convergence of the chain. Chains were considered

667 converged if $\hat{\mathrm{R}}<1.1$.

668

\section{Acknowledgements}

We thank Dora Matkze for sharing the scripts for BEESTS modelling, Sven Bestmann

671 for insightful comments on data, Simon Little for sharing the beta-burst analysis script, Kelsey

672 Sundby for sharing some EEG and EMG data, and Xinze Yu and Hunter Robbins for help in data

673 recording. We gratefully acknowledge our support from NIH: NS106822 and DA026452.

\section{Author contributions}

677 and analyzed the data; S.J., R.H., V.M., and A.R.A wrote the paper.

679 Competing interests

The authors declare no competing financial interests.

\section{Data and scripts}

A core element of this paper is a novel method of calculating single-trial stopping speed

684 from EMG. Accordingly, we provide the EMG and behavioral data from 10 participants in study

685 1, along with analysis scripts, and a brief description of how to execute the scripts

686 (https://osf.io/b2ng5/). Upon acceptance of the paper all EMG, TMS-MEP and EEG data will be 
687 provided at the above link.

\section{References}

690 1. Wagner, J., Makeig, S., Gola, M., Neuper, C. \& Müller-Putz, G. Distinct $\beta$ Band

691 Oscillatory Networks Subserving Motor and Cognitive Control during Gait Adaptation. $J$.

$692 \quad$ Neurosci. 36, 2212-26 (2016).

693 2. Sedgmond, J. et al. Prefrontal brain stimulation during food-related inhibition training:

694 effects on food craving, food consumption and inhibitory control. R. Soc. Open Sci. 6,

$695181186(2019)$.

696 3. Xue, G., Aron, A. R. \& Poldrack, R. A. Common Neural Substrates for Inhibition of

697 Spoken and Manual Responses. Cereb. Cortex 18, 1923-1932 (2008).

698 4. Aron, A. R. The neural basis of inhibition in cognitive control. Neuroscientist 13, 214-228

699 (2007).

700 5. Verbruggen, F. et al. A consensus guide to capturing the ability to inhibit actions and 701 impulsive behaviors in the stop-signal task. Elife 8, (2019).

702 6. Bari, A. \& Robbins, T. W. Inhibition and impulsivity: Behavioral and neural basis of 703 response control. Prog. Neurobiol. 108, 44-79 (2013).

704 7. Alderson, R. M., Rapport, M. D. \& Kofler, M. J. Attention-Deficit/Hyperactivity Disorder 705 and Behavioral Inhibition: A Meta-Analytic Review of the Stop-signal Paradigm. J.

706 Abnorm. Child Psychol. 35, 745-758 (2007).

707 8. Smith, J. L., Mattick, R. P., Jamadar, S. D. \& Iredale, J. M. Deficits in behavioural 708 inhibition in substance abuse and addiction: A meta-analysis. Drug Alcohol Depend. 145, $709 \quad 1-33(2014)$. 
710 9. Lijffijt, M., Kenemans, J. L., Verbaten, M. N. \& van Engeland, H. A Meta-Analytic

711 Review of Stopping Performance in Attention-Deficit/Hyperactivity Disorder: Deficient

712 Inhibitory Motor Control? J. Abnorm. Psychol. 114, 216-222 (2005).

713 10. Snyder, H. R., Kaiser, R. H., Warren, S. L. \& Heller, W. Obsessive-Compulsive Disorder

714 Is Associated With Broad Impairments in Executive Function. Clin. Psychol. Sci. 3, 301-

$715 \quad 330(2015)$.

716 11. Lavagnino, L., Arnone, D., Cao, B., Soares, J. C. \& Selvaraj, S. Inhibitory control in

717 obesity and binge eating disorder: A systematic review and meta-analysis of

718 neurocognitive and neuroimaging studies. Neurosci. Biobehav. Rev. 68, 714-726 (2016).

719 12. Aron, A. R., Robbins, T. W. \& Poldrack, R. A. Inhibition and the right inferior frontal

720 cortex: One decade on. Trends Cogn. Sci. 18, 177-185 (2014).

721 13. Schall, J. D. \& Godlove, D. C. Current advances and pressing problems in studies of 722 stopping. Curr. Opin. Neurobiol. 22, 1012-1021 (2012).

723 14. Casey, B. J. et al. The Adolescent Brain Cognitive Development (ABCD) study: Imaging acquisition across 21 sites. Dev. Cogn. Neurosci. 32, 43-54 (2018).

725 15. Skippen, P. et al. Reliability of triggering inhibitory process is a better predictor of 726 impulsivity than SSRT. Acta Psychol. (Amst). 192, 104-117 (2019).

727 16. Lijffijt, M. et al. Differences between low and high trait impulsivity are not associated 728 with differences in inhibitory motor control. J. Atten. Disord. 8, 25-32 (2004).

729 17. Chowdhury, N. S., Livesey, E. J., Blaszczynski, A. \& Harris, J. A. Pathological Gambling 730 and Motor Impulsivity: A Systematic Review with Meta-Analysis. J. Gambl. Stud. 33, $731 \quad$ 1213-1239 (2017).

732 18. Friedman, N. P. \& Miyake, A. The Relations Among Inhibition and Interference Control 
Functions: A Latent-Variable Analysis. J. Exp. Psychol. Gen. 133, 101-135 (2004).

734 19. McLaughlin, N. C. R. et al. Stop Signal Reaction Time Deficits in a Lifetime ObsessiveCompulsive Disorder Sample. J. Int. Neuropsychol. Soc. 22, 785-789 (2016).

736 20. Enkavi, A. Z. et al. Large-scale analysis of test-retest reliabilities of self-regulation measures. Proc. Natl. Acad. Sci. U. S. A. 116, 5472-5477 (2019).

738 21. Bissett, P., Poldrack, R. \& Logan, G. D. Severe violations of independence in response 739 inhibition tasks are pervasive and consequential. doi:10.31234/OSF.IO/KPA65

740 22. Raud, L. \& Huster, R. J. The Temporal Dynamics of Response Inhibition and their 741 Modulation by Cognitive Control. Brain Topogr. 30, 486-501 (2017).

742 23. Coxon, J. P., Stinear, C. M. \& Byblow, W. D. Intracortical Inhibition During Volitional 743 Inhibition of Prepared Action. J. Neurophysiol. 95, 3371-3383 (2006).

744 24. van den Wildenberg, W. P. M. et al. Mechanisms and Dynamics of Cortical Motor 745 Inhibition in the Stop-signal Paradigm: A TMS Study. J. Cogn. Neurosci. 22, 225-239 $746 \quad(2010)$

747 25. Badry, R. et al. Suppression of human cortico-motoneuronal excitability during the Stop$748 \quad$ signal task. Clin. Neurophysiol. 120, 1717-1723 (2009).

749 26. Wessel, J. R., Reynoso, H. S., Aron, A. R., Sequoyah Reynoso, H. \& Aron, A. R. Saccade 750 suppression exerts global effects on the motor system. J. Neurophysiol. 110, 883-90 (2013).

752 27. Cai, W., Oldenkamp, C. L. \& Aron, A. R. Stopping speech suppresses the task-irrelevant 753 hand. Brain Lang. 120, 412-5 (2012).

754 28. Wessel, J. R. \& Aron, A. R. Unexpected events induce motor slowing via a brain 755 mechanism for action-stopping with global suppressive effects. J. Neurosci. Off. J. Soc. 
Neurosci. 33, 18481-18491 (2013).

757

758

759

760

761

762

763

764

765

766

767

768

769

770

771

772

773

774

775

776

777

778

29. Wessel, J. R. et al. Stop-related subthalamic beta activity indexes global motor suppression in Parkinson's disease. Mov. Disord. 31, 1846-1853 (2016).

30. Wessel, J. R. \& Aron, A. R. On the Globality of Motor Suppression: Unexpected Events and Their Influence on Behavior and Cognition. Neuron 93, 259-280 (2017).

31. Swann, N. et al. Intracranial EEG reveals a time- and frequency-specific role for the right inferior frontal gyrus and primary motor cortex in stopping initiated responses. $J$. Neurosci. 29, 12675-85 (2009).

32. Wagner, J., Wessel, J. R., Ghahremani, A. \& Aron, A. R. Establishing a Right Frontal Beta Signature for Stopping Action in Scalp Electroencephalography: Implications for Testing Inhibitory Control in Other Task Contexts. J. Cogn. Neurosci. 1-12 (2017). doi:10.1162/jocn_a_01183

33. Castiglione, A., Wagner, J., Anderson, M. \& Aron, A. R. Preventing a Thought from Coming to Mind Elicits Increased Right Frontal Beta Just as Stopping Action Does. Cereb. Cortex 29, 2160-2172 (2019).

34. Mcgarry, T., Inglis, J. T. \& Franks, I. M. Against a Final Ballistic Process in the Control of Voluntary Action: Evidence Using the Hoffmann Reflex. Motor Control 4, 469-485 (2000).

35. Little, S., Bonaiuto, J., Barnes, G. \& Bestmann, S. Motor cortical beta transients delay movement initiation and track errors. bioRxiv 384370 (2018). doi:10.1101/384370

36. Matzke, D., Love, J. \& Heathcote, A. A Bayesian approach for estimating the probability of trigger failures in the stop-signal paradigm. Behav. Res. Methods 49, 267-281 (2017).

37. Shin, H., Law, R., Tsutsui, S., Moore, C. I. \& Jones, S. R. The rate of transient beta 
frequency events predicts behavior across tasks and species. Elife 6, 1-31 (2017).

780 38. Bell, A. J. \& Sejnowski, T. J. An Information-Maximization Approach to Blind

Separation and Blind Deconvolution. Neural Comput. 7, 1129-1159 (1995).

782

39. Bellumori, M., Jaric, S. \& Knight, C. A. The rate of force development scaling factor (2011).

40. De Jong, R., Coles, M. G., Logan, G. D. \& Gratton, G. In search of the point of no return: the control of response processes. J. Exp. Psychol. Hum. Percept. Perform. 16, 164-182

41. Jana, S. \& Murthy, A. Task context determines whether common or separate inhibitory signals underlie the control of eye-hand movements. https://doi.org/10.1152/jn.00085.2018 (2018). doi:10.1152/JN.00085.2018

791 42. Hanes, D. P., Patterson, W. F. \& Schall, J. D. Role of frontal eye fields in countermanding saccades: visual, movement, and fixation activity. J. Neurophysiol. 79, 817-834 (1998).

793 43. Ogasawara, T., Nejime, M., Takada, M. \& Matsumoto, M. Primate Nigrostriatal Dopamine System Regulates Saccadic Response Inhibition. Neuron 100, 1513-1526.e4 (2018).

796 44. Osada, T. et al. An essential role of the intraparietal sulcus in response inhibition predicted by parcellation-based network. J. Neurosci. 39, 2244-18 (2019).

798 45. Wessel, J. R. \& Aron, A. R. It's not too late: The onset of the frontocentral P3 indexes successful response inhibition in the stop-signal paradigm. Psychophysiology 52, 472-480 (2015).

801 46. Clark, L. et al. Association Between Response Inhibition and Working Memory in Adult 
ADHD: A Link to Right Frontal Cortex Pathology? Biol. Psychiatry 61, 1395-1401

(2007).

804 47. Kalanthroff, E. et al. The Role of Response Inhibition in Medicated and Unmedicated

805 Obsessive-Compulsive Disorder Patients: Evidence from the Stop-Signal Task. Depress.

$806 \quad$ Anxiety 34, 301-306 (2017).

807 48. Lipszyc, J. \& Schachar, R. Inhibitory control and psychopathology: A meta-analysis of studies using the stop signal task. J. Int. Neuropsychol. Soc. 16, 1064-1076 (2010).

49. Rossi, S., Hallett, M., Rossini, P. M. \& Pascual-Leone, A. Screening questionnaire before TMS: An update. Clin. Neurophysiol. 122, 1686 (2011).

811 50. Brainard, D. H. The Psychophysics Toolbox. Spat. Vis. 10, 433-436 (1997).

812 51. Verbruggen, F. \& Logan, G. D. Models of response inhibition in the stop-signal and stop813 change paradigms. Neurosci. Biobehav. Rev. 33, 647-661 (2009).

814 52. Hamada, M., Murase, N., Hasan, A., Balaratnam, M. \& Rothwell, J. The role of interneuron networks in driving human motor cortical plasticity. Cereb. Cortex 23, 15931605 (2013).

817 53. Hannah, R. \& Rothwell, J. Pulse Duration as Well as Current Direction Determines the Specificity of Transcranial Magnetic Stimulation of Motor Cortex during Contraction. Brain Stimul. 10, 106-115 (2017).

820 54. Rossini, P. M. et al. Non-invasive electrical and magnetic stimulation of the brain, spinal $821 \quad$ cord, roots and peripheral nerves: Basic principles and procedures for routine clinical and research application: An updated report from an I.F.C.N. Committee. Clinical Neurophysiology 126, 1071-1107 (2015).

824 55. Delorme, A. \& Makeig, S. EEGLAB: an open source toolbox for analysis of single-trial 
EEG dynamics including independent component analysis. J. Neurosci. Methods 134, 9$21(2004)$

827 56. Oostenveld, R. \& Oostendorp, T. F. Validating the boundary element method for forward

828 and inverse EEG computations in the presence of a hole in the skull. Hum. Brain Mapp.

$829 \quad 17,179-192(2002)$.

830 57. Matzke, D., Dolan, C. V., Logan, G. D., Brown, S. D. \& Wagenmakers, E.-J. J. Bayesian

831 parametric estimation of stop-signal reaction time distributions. J. Exp. Psychol. Gen. 142,

$832 \quad 1047-1073(2013)$

833

834 